\title{
Use of an EHR-Integrated Point-of-Care Mobile Medical Photography Application in a Pediatric Emergency Department
}

\author{
${ }^{1}$ Mayo Clinic Alix School of Medicine, Mayo Clinic, Rochester, \\ Minnesota, United States \\ 2 Division of Pediatric Hematology/Oncology, Department of \\ Pediatric and Adolescent Medicine, Mayo Clinic, Rochester, \\ Minnesota, United States \\ ${ }^{3}$ Division of Biomedical Statistics and Informatics, Mayo Clinic, \\ Rochester, Minnesota, United States \\ ${ }^{4}$ Department of Emergency Medicine, Mayo Clinic, Rochester, \\ Minnesota, United States \\ ${ }^{5}$ Department of Pediatric and Adolescent Medicine, Mayo Clinic, \\ Rochester, Minnesota, United States
}

Richmond M. Castillo ${ }^{1}$ Grace Y. Kim ${ }^{1} \quad$ Kirk D. Wyatt ${ }^{2}$ Christine M. Lohse ${ }^{3}$ Thomas R. Hellmich ${ }^{4,5}$

Appl Clin Inform 2019;10:888-897.
Address for correspondence Thomas R. Hellmich, MD, Department of Emergency Medicine and Pediatric and Adolescent Medicine, Mayo Clinic, 200 First Street Southwest, Rochester, Minnesota 55905, United States (e-mail: Hellmich.Thomas@mayo.edu).

\section{Abstract}

Keywords

- medical photography

- photography

- emergency medicine

- mobile application

- pediatric emergency medicine
Background Mobile applications allow health care providers to capture point-of-care medical photographs and transfer them to the electronic health record (EHR). It is unclear how providers use these photographs or how they affect clinical care.

Objectives We aimed to understand the content, purpose, and outcomes of point-ofcare medical photography performed in the pediatric emergency department (ED) at large academic medical center.

Methods A retrospective chart review was conducted of patients $<21$ years of age who were seen in the ED and photographed between March 29, 2015 and July 1, 2017 using a secure smartphone application integrated with the EHR. Inter-rater agreement and reliability between the two reviewers was assessed for the first 50 charts, and any discrepancies in interpretation were resolved before proceeding with the remaining data abstraction. The documented rationale for photography, content of photographs, and outcomes were recorded.

Results We identified 619 clinical encounters involving photographs of 605 patients who were eligible for inclusion. Skin was photographed in 499 (81\%). The most common finding was rash $(N=177 ; 29 \%)$. Photos were of acceptable quality, with $569(94 \%)$ achieving a score between 4 and 5 out of 5 . The primary use of photography was documentation $(N=334 ; 54 \%)$, though teleconsultation was noted in 38 (6\%). Nearly one-third $(N=187$; $30 \%$ ) of patients were seen in the ED or outpatient clinic for any reason within 2 weeks, and in 25 (13\%), clinical notes explicitly referenced the initial photograph(s). In 53 (9\%) cases, patients were photographed at a clinical visit in the subsequent 2 weeks, suggesting that photography was used to track changes over time.

Conclusion Documentation of findings using mobile point-of-care photography allows for high-fidelity documentation and facilitates continuity of care. received

July 15, 2019

accepted after revision

September 26, 2019 (c) 2019 Georg Thieme Verlag KG Stuttgart · New York
DOI https://doi.org/

10.1055/s-0039-1700870.

ISSN 1869-0327. 


\section{Background and Significance}

Medical photography has a longstanding role for accurately documenting clinical findings. Since the earliest known clinical photograph was taken in the $1840 \mathrm{~s},{ }^{1}$ the practice has evolved significantly. Many institutions-including our own -employ full-time medical photographers who utilize professional-grade photography equipment to capture high-fidelity photographs of clinical findings. However, the process does not necessarily need to be so sophisticated. Hospitals and clinics may purchase a low-cost digital camera that is stored in a centralized location and is used by clinical staff to capture photographs. Prior to the advent of digital cameras, instant (e.g., Polaroid) cameras were well-suited for this purpose. Today, digital cameras and cameras integrated within mobile devices fulfill this role. Medical photography has been used in a variety of specialties, including emergency medicine, dentistry, surgical specialties, and dermatology. ${ }^{2-10}$

\section{Point-of-Care Medical Photographs and Integration with the Electronic Health Record}

As is the case when any new technology is used in health care, use of digital cameras to capture patient photographs introduces practical, ethical, and legal issues that must be carefully considered. From a practical standpoint, digital cameras may not easily interface with the electronic health record (EHR), thereby presenting a challenge when the time comes to integrate photographs with the rest of the patient's medical records. While affixing an instant-develop photograph to the paper medical record using a staple was an easy task, lack of interoperability is a key barrier to integrating digital photographs within the EHR. From an ethical and legal standpoint, photographs may contain sensitive and personally-identifiable information. Medical recordkeeping laws (e. g., Health Insurance Portability and Accountability Act of 1996 [HIPAA]) require that patients have access to their medical records, which can include photographs. ${ }^{11}$ Furthermore, health care providers must protect individually identifiable health information and enact safeguards to protect personal health information stored in electronic form. Procedures for medical photography that do not take these considerations into account risk violating basic patient rights and may expose providers and institutions to legal liability. Although patients' acceptance of smartphone-based medical photography is increased when they are provided assurances that photography is conducted using a secure application, ${ }^{7}$ the literature suggests that dedicated, secure applications integrated with the EHR are used in a minority of cases. ${ }^{8,9}$

In light of the challenges associated with handling of digital patient photographs, institutions such as Brigham and Women's hospital ${ }^{12}$ and the NHS Trust ${ }^{10}$ have transitioned from ad hoc methods to integrate photographs into the medical record to the adoption of a dedicated, secure mobile application which runs on a user's smartphone. ${ }^{13}$ In March 2015, Mayo Clinic similarly released such an application ("PhotoExam") which runs on the iOS platform (Apple Inc., Cupertino, CA) for internal use. We previously published our experience in the first 8 months of use, which encom- passed a variety of users in terms of clinical specialty and work role. ${ }^{14}$ We also described its use in primary care, where over one-quarter of point-of-care medical photography sessions using a mobile device were associated with teleconsultation with a dermatologist. ${ }^{15}$ However, uses within specialties outside of primary care, the intended purpose for capturing photographs and clinical outcomes following medical photography remain unclear. As with any practice innovation, evidence of clinical impact is important to justify ongoing support and expansion of point-of-care photography applications and to identify practice gaps encountered during their use.

\section{Objectives}

To supplement our anecdotal experience and previous research, we aimed to systematically describe the purpose and outcomes of a point-of-care clinical photography application for evaluation and management of pediatric patients in the emergency department (ED) at a large academic medical center. Our objectives were to (1) understand reasons why providers capture medical photographs using the PhotoExam application; (2) tabulate what types of findings were photographed using the application; and (3) assess follow-up related outcomes of patients photographed using the application.

\section{Methods}

\section{PhotoExam Application}

The functionality of the PhotoExam application is described elsewhere. ${ }^{14}$ The application is available to all clinical staff with patient care responsibilities at our institution. Online training resources provided technical support on use but did not provide practical guidance on clinical situations where photography may be useful or advice on how to take high-quality photographs. After logging in and loading a patient record, the user was required to toggle a button indicating that consent has been obtained ( - Fig. 1). This toggle button triggers a hard stop popup, which requires the user to attest that all institutional procedures and standards for obtaining consent have been followed. Departmental policies varied in terms of the form of consent required (i.e., written vs. verbal). If patient consent is not affirmed using this two-step process, the user is not permitted to capture photographs using the application.

After consent has been obtained, users are prompted to tag the anatomical site that is being photographed and are allowed to take up to six photographs of each anatomical site of interest per session using the camera built into the mobile device. Users can also include a brief text description to accompany the photographs. Photographs are then uploaded to the patient's medical record. Images are stored within an internal Digital Imaging and Communications in Medicine compliant digital clinical asset management system. Following successful upload or exit from the application, the photographs are permanently deleted from the user's device. Photographs are available in the EHR within minutes of upload for any provider with EHR access to view. Images are viewable from within the internally-developed image 


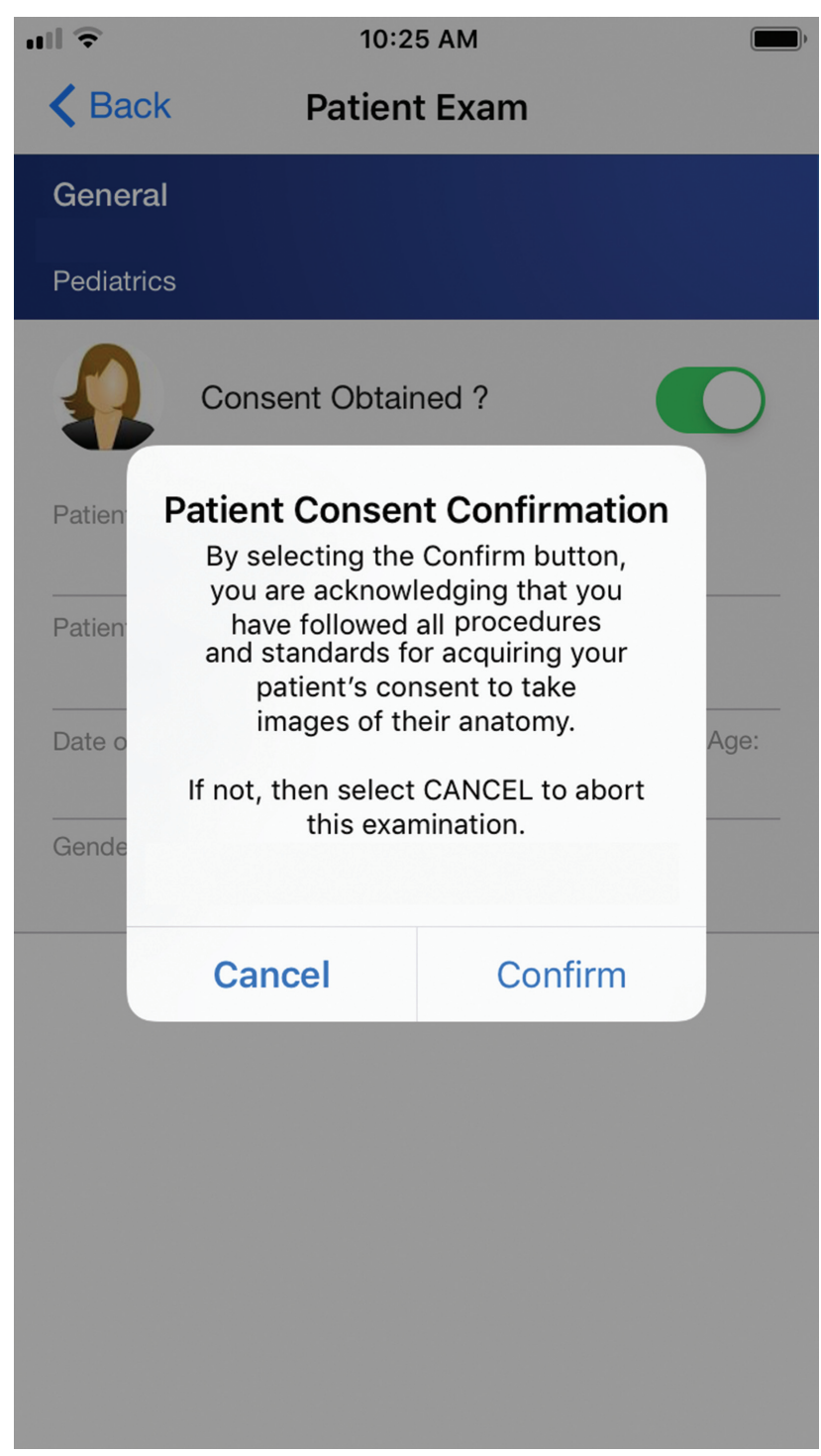

Fig. 1 Two-step patient consent verification process. After loading a patient record, the user must toggle the green button (top right), which prompts an interruptive popup alert requiring the user to attest the consent has been obtained following institutional procedures and standards.

viewing component of the EHR that is used across the institution to view all radiographs and clinical photographs. This software can be launched from the third-party EHR used at our institution (Epic, Epic Systems Corporation, Verona, WI) in a manner that hands off patient context from one application to the other.

\section{Identification of Records}

The PhotoExam photograph database was queried to identify all patients who were photographed using the PhotoExam application between March 29, 2015 and July 1, 2017. To identify patients who were photographed in the pediatric $E D$, we cross referenced all patients photographed using the application with patients $<21$ years of age who were seen on the same date in the ED. We excluded patients who declined consent to use of their records for research purposes. Photographs and clinical documentation within the EHR were reviewed to determine if the photographs on the date indicated actually corresponded to an ED visit and were taken by the members of the ED team. Photographs that corresponded to different settings (e.g., inpatient hospitalization, other same-day outpatient clinical encounter) or other teams (e.g., specialist saw patient for in-person consultation in ED and captured photographs) were excluded.

\section{Chart Review}

Clinical notes in the EHR were reviewed to ascertain the documented rationale for and outcomes of taking photographs. In some cases, the documented rationale was made explicit (e.g., clinical note indicates that photographs were taken for future reference of another provider) and in some cases it was inferred (e.g., provider did not document specific rationale for photography but indicated that they called a specialist to review the photographs with them remotely). If the reviewers could not determine the purpose of photography with confidence, they indicated so and did not speculate on the purpose. A review of notes of subsequent clinical encounters relating to the same chief complaint was also conducted to determine if the photographs taken were referenced at later visits. Photographs themselves were reviewed to assess their content and quality. Subsequent photographs taken at other clinical encounters were also assessed to determine whether they demonstrated the finding initially photographed. Clinical documentation and signed consent forms were used to assess the method of consent used for medical photography. Records were also reviewed to determine other aspects of the patient's care, including timestamps for arrival and disposition, which allowed calculation of length of stay.

A subset of 50 randomly-selected records were independently reviewed in duplicate by two reviewers (R.M.C., G.Y.K.) to measure inter-rater agreement and reliability. A convenience sample size of 50 was selected to allow for sufficient diversity in the purposes for photography encountered by the two reviewers, while still being feasible to complete in a timely manner. Conflicts within this subset were resolved by consensus when possible and by arbitration by a third party when consensus could not be achieved. Both reviewers were senior medical students. The reviewers were not blinded to the purpose and goals of the study and did not receive any specialized training in advance of the study. After assessing inter-rater agreement and reliability and discussing the reasons for discrepancies in the reviewed records, we proceeded to review the remaining records with one reviewer reviewing each record.

Quality was assessed using a five-point rubric that has been previously described. ${ }^{14}$ Photographs were assessed for quality according to whether (1) the photographed location matched the tagged metadata, (2) the photograph clearly showed the area of interest, (3) the photograph portrayed size, (4) the image lacked discoloration, and (5) the image lacked blurriness. A "quality score" was calculated as the percentage of criteria that were met out of a total of five.

Data were collected in RedCap and then exported for analysis. ${ }^{16}$ 
Table 1 Patient demographics and encounter characteristics

\begin{tabular}{|l|l|}
\hline & Mean (SD) \\
\hline Age at visit (years) & $7.8(6.3)$ \\
\hline & Median (IQR) \\
\hline Photographs captured at visit & $3(2-4)$ \\
\hline Length of stay (hours) & $2.5(1.5-3.7)$ \\
\hline Sex & $n(\%)$ \\
\hline Female & $302(49)$ \\
\hline Male & $317(51)$ \\
\hline Race $(N=615)$ & \\
\hline White & $471(77)$ \\
\hline Non-white & $144(23)$ \\
\hline
\end{tabular}

Abbreviations: IQR, interquartile ranges; SD, standard deviation.

\section{Statistical Analysis}

Continuous features were summarized with means and standard deviations (SD) when approximately normally distributed and with medians and interquartile ranges (IQRs) otherwise; categorical features were summarized with frequency counts and percentages. Agreement and reliability were summarized using percent agreement (overall, positive, and negative agreement) and kappa statistics, respectively, with $95 \%$ confidence intervals (CIs). ${ }^{17,18}$ Statistical analyses were performed using SAS version 9.4 (SAS Institute; Cary, NC).

\section{Results}

\section{Patient Demographics}

We identified 619 encounters involving 605 distinct patients who were eligible for inclusion. The mean patient age was 7.8 (SD 6.3) years ( - Table 1). There was an equal proportion of male and female patients $(N=317 ; 51 \%$ male $)$ and most $(N=471 ; 77 \%)$ patients were white $(-$ Table 1$)$.

\section{Encounter Demographics}

Patients were photographed by 96 unique providers. Sixtythree (66\%) providers contributed between one and three visits, with the remainder contributing more. The median number of photographs per encounter was 3 (IQR 2-4), and median length of stay was 2.5 (IQR 1.5-3.7) hours. All photographers were physicians, medical students, or physician assistants. Attending physicians accounted for the majority ( $N=316 ; 51 \%)$ of photographs, followed by pediatrics residents $(N=192 ; 31 \%)$ and emergency medicine residents $(N=88 ; 14 \%)$ rotating in the ED.

\section{Inter-Rater Agreement and Reliability}

The reviewers were asked whether each photograph was eligible for inclusion in the study. Of the 50 randomlyselected records reviewed, the reviewers agreed that the photograph should be included for 31, agreed that the photograph should not be included for 6 , and disagreed for 13 , resulting in overall agreement of $74 \%$ (95\% CI 62-86), positive agreement of $83 \%$ (95\% CI 73-92), negative agreement of $48 \%$ ( $95 \%$ CI $24-72$ ), and a kappa statistic of 0.36 (95\% CI 0.13-0.59). Inter-rater agreement and reliability for documented purposes of photography and quality rubric items for the 31 records that both reviewers agreed were eligible for inclusion are summarized in -Table 2 . For some items with a high level of agreement, the kappa statistic was paradoxically zero as a result of the variables rarely being selected. ${ }^{19}$ For this reason, percent positive agreement and percent negative agreement are also reported. As an example, overall agreement for the documented purpose of photography of "to send to specialist for management advice" was $97 \%$, but the kappa statistic was 0 because this documented purpose was not selected for 30 of the 31 records reviewed; for the remaining record, one reviewer selected this purpose, but the other did not. As such, positive agreement, or the percentage that one of the reviewers selected this purpose given that the other reviewer did as well, was $0 \%$, while negative agreement, which represents a similar conditional percentage for not selecting this purpose, was $98 \%$.

\section{Content of Photographs}

Manual review of photographs revealed that 499 (81\%) were of skin. The remaining anatomical areas accounted for $4 \%$ or fewer of photographs. Unexpected photographed findings included medical devices or items external to the patient (e. g., foreign body; $N=36$ ). The most common findings in photographs were rash $(N=177 ; 29 \%)$, infection $(N=136$; $22 \%)$, and penetrating trauma $(N=114 ; 18 \%)$. Remaining findings each accounted for $7 \%$ or fewer encounters and are included in -Table 3 .

\section{Consent}

Explicit documentation of consent within the EHR could be identified in only 226 (37\%) cases. In 67 (30\%) of these, written consent was documented in the medical record; in $24(11 \%)$, the clinical note indicated verbal consent was obtained; in 135 (60\%), the note reflected that consent was obtained, but the method of consent was not explicitly specified and was presumed to be verbal because a signed written consent form could not be located in the medical record.

\section{Photograph Quality}

Using a previously-developed five-point rubric, photographs were generally deemed to be of sufficient quality for clinical purposes. One-third ( $N=203 ; 33 \%)$ attained a perfect score, and 366 (60\%) were assigned a score of four out of five, with the most commonly missed item on the quality rubric being portrayal of the size of the finding(s) $(N=220 ; 36 \%$ portrayed size).

\section{Purpose of Photography}

The purpose of photography was challenging to determine with confidence, as it was only explicitly documented in notes of 102 (16\%) cases. In 296 (48\%) cases, reviewers felt confident determining the implied purpose based on the context provided in the note, and in 221 (36\%), reviewers 
Table 2 Inter-rater agreement and reliability, $N=31$

\begin{tabular}{|c|c|c|c|c|}
\hline Item & $\begin{array}{l}\text { Percent agreement } \\
\text { overall }(95 \% \mathrm{Cl})\end{array}$ & $\begin{array}{l}\text { Percent positive } \\
\text { agreement }(95 \% \mathrm{CI})\end{array}$ & $\begin{array}{l}\text { Percent negative } \\
\text { agreement }(95 \% \mathrm{Cl})\end{array}$ & Карра (95\% CI) \\
\hline \multicolumn{5}{|l|}{$\begin{array}{l}\text { Documented purposes of } \\
\text { photography (yes vs. no) }\end{array}$} \\
\hline $\begin{array}{l}\text { To send to specialist for } \\
\text { assistance with diagnosing } \\
\text { a condition. }\end{array}$ & $97(91-100)$ & $67(5-100)$ & $98(95-100)$ & $0.65(0.02-1)$ \\
\hline $\begin{array}{l}\text { To send to a specialist for } \\
\text { management advice when the } \\
\text { diagnosis is known by the } \\
\text { photographing provider. }\end{array}$ & $97(91-100)$ & $0(0-0)$ & $98(95-100)$ & $0(0-0)$ \\
\hline $\begin{array}{l}\text { To facilitate in-person specialist } \\
\text { consultation in the emergency } \\
\text { department. }\end{array}$ & $100(100-100)$ & $0(0-0)$ & $100(100-100)$ & $1(1-1)$ \\
\hline $\begin{array}{l}\text { To document changes over time } \\
\text { with photographs of the same } \\
\text { patient taken at different times. }\end{array}$ & $97(91-100)$ & $0(0-0)$ & $98(95-100)$ & $0(0-0)$ \\
\hline $\begin{array}{l}\text { To document the appearance } \\
\text { of the photographed area for } \\
\text { reference of a provider who } \\
\text { may see the patient in the } \\
\text { future (e.g., if the findings were } \\
\text { to worsen or not improve). }\end{array}$ & $94(85-100)$ & $50(0-100)$ & $97(92-100)$ & $0.47(-0.12$ to 1$)$ \\
\hline $\begin{array}{l}\text { To document the examination } \\
\text { more vividly that the provider } \\
\text { could describe in words. }\end{array}$ & $81(67-95)$ & $86(76-97)$ & $67(42-92)$ & $0.53(0.21-0.86)$ \\
\hline $\begin{array}{l}\text { So that the examination did } \\
\text { not have to be repeated by } \\
\text { multiple providers. }\end{array}$ & $100(100-100)$ & $0(0-0)$ & $100(100-100)$ & $1(1-1)$ \\
\hline For educational purposes. & $100(100-100)$ & $0(0-0)$ & $100(100-100)$ & $1(1-1)$ \\
\hline Purpose not specified. & $84(71-97)$ & $62(30-93)$ & $90(81-99)$ & $0.52(0.16-0.88)$ \\
\hline \multicolumn{5}{|l|}{$\begin{array}{l}\text { Quality rubric (yes vs. no vs. } \\
\text { unknown) }\end{array}$} \\
\hline $\begin{array}{l}\text { Does the location in the photos } \\
\text { match the metadata entered } \\
\text { by the user? }\end{array}$ & $94(85-100)$ & $97(92-100)$ & $0(0-0)^{a}$ & $0(0-0)$ \\
\hline $\begin{array}{l}\text { Do the photos clearly show } \\
\text { where the area of interest is on } \\
\text { the body? }\end{array}$ & $97(91-100)$ & $98(95-100)$ & $0(0-0)$ & $0(0-0)$ \\
\hline $\begin{array}{l}\text { Do the photos portray the size by } \\
\text { including nearby landmarks on } \\
\text { the body for comparison and/or } \\
\text { ruler next to the area of interest? }\end{array}$ & 87 (75-99) & $83(67-99)$ & $89(79-100)$ & $0.73(0.50-0.97)$ \\
\hline $\begin{array}{l}\text { Are the images discolored to } \\
\text { the point of affecting } \\
\text { documentation? }\end{array}$ & $100(100-100)$ & $0(0-0)$ & $100(100-100)$ & $1(1-1)$ \\
\hline $\begin{array}{l}\text { Does image quality (e.g., blur- } \\
\text { riness) limit the ability to as- } \\
\text { sess the area of interest? }\end{array}$ & $100(100-100)$ & $0(0-1)$ & $100(100-100)$ & $1(1-1)$ \\
\hline
\end{tabular}

Abbreviation: $\mathrm{Cl}$, confidence interval.

${ }^{a}$ For this feature, no reviewer selected "no." One reviewer selected "unknown" for two records. As such, the column for percent negative agreement represents percent unknown agreement. The reviewers selected "yes" or "no" only for all other items in the quality rubric.

could not determine the purpose of photography with confidence. A majority of photographs ( $54 \% ; N=334$ ) appeared to be captured to simply document the examination for documentation purposes. Other purposes included documenta- tion for explicit future reference $(N=29 ; 5 \%)$ as well as storeand-forward teleconsultation for diagnostic recommendations ( $N=12 ; 2 \%)$ or management $(N=26 ; 4 \%)$ recommendations. Qualitative review revealed common patterns of 
Table 3 Content of photographs

\begin{tabular}{|l|l|}
\hline Finding & $N(\%)$ \\
\hline Rash & $177(29)$ \\
\hline Infection & $136(22)$ \\
\hline Penetrating trauma & $114(18)$ \\
\hline Nonpenetrating trauma & $41(7)$ \\
\hline Swelling/edema & $27(4)$ \\
\hline Burn & $26(4)$ \\
\hline Genital complaint & $20(3)$ \\
\hline Patient/parent brought item & $10(2)$ \\
\hline External object & $9(1)$ \\
\hline Wound or surgical site & $8(1)$ \\
\hline Bug bite/sting & $6(1)$ \\
\hline Neurologic finding & $6(1)$ \\
\hline Ulcer & $5(1)$ \\
\hline Foreign body & $2(<1)$ \\
\hline Other & $52(8)$ \\
\hline
\end{tabular}

reference to photographs and their rationale. Commonly, the presence of supplementary photographs would be simply noted in the "History of Present Illness" section or documented in the "Physical Examination" section of clinical notes alongside a textual description. In other cases, photographs were noted in the "Impression, Report and Plan" section along with an indication that a specialist has reviewed or will review the photographs.

\section{Outcomes}

In $134(22 \%)$ cases, at least one specialist saw the patient while in the ED. In 61 (10\%), consultation was remotely obtained by phone from a specialist. Specialists most likely to see patients in the ED were orthopedic surgeons $(N=18$; $13 \%$ of in-person consultations), dermatologists $(N=16$; $12 \%)$, and otolaryngologists $(N=13 ; 10 \%)$, with all other specialties representing $7 \%$ or less. Dermatology provided the majority $(N=36 ; 59 \%)$ of remote consultations by phone with all other specialties representing $7 \%$ or less.

In 187 (30\%) cases, patients were seen in the ED or at an outpatient visit within 2 weeks of their initial visit for any reason, and in $25(13 \%)$ of these cases, the photographs captured in the initial visit were referenced in the note. Furthermore, in 15 (2\%) cases, the photographed clinical finding had been photographed by another provider in the preceding 2 weeks, and in $53(9 \%)$ cases, the clinical finding was photographed during a clinical encounter in the subsequent 2 weeks, indicating that photographs were used to track changes over time. Qualitative review of these photographs revealed common use cases where multiple photographs were taken over time. Some photographs captured at additional time points appeared to focus on different aspects of the finding (e.g., different zoom/magnification level or angle), some photographs replicated the same findings but with higher fidelity (e.g., professional medical photography), some demonstrated the appearance before and after an intervention (e.g., surgery), and some tracked evolution of findings over time (e.g., natural evolution or response to therapy).

\section{Discussion}

\section{Primary Findings}

Mobile point-of-care medical photography was widely used in our pediatric ED. Most visits included between two and four photographs of the area(s) of interest. Photographs as a whole were of high quality. Unsurprisingly, skin findings accounted for the vast majority of photographs, including rashes, infectious skin findings, and penetrating trauma.

Consent was not well-documented in the medical record; however, verbal consent appeared to predominate. The reasons why consent was poorly documented were unclear and could not be determined using the retrospective study design; prospective surveys or focus groups may yield additional insights regarding providers' consent practices. When the purpose of photography could be determined, the most common purpose was simply for clinical documentation; however, providers rarely documented the explicit rationale for taking photographs. The reasons for this, also, could not be determined with a retrospective study design. It is possible that providers thought that the rationale was self-evident or that specific documentation of the rationale was not necessary to facilitate patient care. The latter possibility is relevant because providers who capture photographs may use them for different reasons compared with providers who view them. Nearly one-third of patients were seen at followup visits for the same complaint during the following 2 weeks, and the photographs taken in the ED were referenced at $13 \%$ of these visits. We observed cases where photographs were taken at multiple visits to track findings over time.

\section{Comparison to Other Studies}

To our knowledge, this study is the first to comprehensively assess the content, purpose, and downstream outcomes of point-of-care medical photography conducted using a mobile device. A small pilot survey of 100 patients seen an ED in the United Kingdom, which referred to medical photography in general (i.e., not referring specifically relating to photography using a mobile device), observed that $84 \%$ of the patients would consent to being photographed for purposes of medical education. ${ }^{2}$ Furthermore, a report from the United Kingdom reported that photographic documentation of open fractures increased following implementation of a similar secure, EHR-integrated mobile point-of-care clinical photography application. ${ }^{10}$

Our previous study on use of the PhotoExam application in primary care specialties at Mayo Clinic demonstrated that teleconsultation was used in approximately one-quarter of cases where the PhotoExam application was used. ${ }^{15}$ In contrast, we observed in the present study teleconsultation in the ED was surprisingly rare. It is possible that we 
underestimated actual teleconsultation utilization because we were limited to retrospective review of medical records and teleconsultation may not be well-documented. In $22 \%$ of the cases photographed by the emergency medicine team, at least one specialist saw the patient for in-person consultation in the ED. The authors' anecdotal experience suggests that in many cases, the primary emergency medicine team captures photographs prior to calling consulting services and reviews photographs with them over the phone before they see the patient in-person. Therefore, we hypothesize that teleconsultation often precedes in-person consultation but may not be fully captured in clinical documentation.

We observed a low rate of documentation of verbal consent within the medical record; however, this is consistent with consent documentation rates reported in other similar studies. A small Australian study of 13 dermatology registrars reported that $92 \%$ obtained verbal consent when capturing photographs of a patient using a smartphone, yet only $15 \%$ consistently documented verbal consent in the medical record. ${ }^{6}$ Another Australian study of 105 dermatologists reported that written consent was obtained by $2 \%$, verbal consent was obtained and documented in the record by $30 \%$, verbal consent was obtained but not documented anywhere by $46 \%$, and $13 \%$ did not expressly obtain patient consent (the remaining 9\% did not utilize smartphones to capture clinical photographs). ${ }^{18}$ Similarly, a study on attitudes of Canadian plastic surgeons observed that $75 \%$ felt that verbal consent was sufficient but did not report the proportion which explicitly documented that consent in the medical record. ${ }^{8}$

\section{Implications for Clinical Care}

Point-of-care medical photography has the potential to become a new standard of care, given the ease at which high-fidelity photographs can be captured and reviewed by clinicians. Although photography applications facilitate formal or informal (i.e., "curbside") teleconsultations with specialists without the need for the specialist to see the patient in-person, our anecdotal experience has been that store-and-forward teleconsultation also helps specialists triage in-person consultations. For example, one of the coauthors shared a photograph of a severe dog bite with the oncall plastic surgeon who subsequently alerted the operating room staff of the need for an emergent add-on procedure prior to seeing the patient in person. This call was noted to expedite the time needed to transfer the patient to the operating room. In the time-pressured environment of over-crowded EDs, interventions that shorten the overall length of stay and time to disposition are welcomed. Unfortunately, given the nature of our study and challenges inherent in identifying a suitable control group, we were not able to systematically assess whether photography decreased length of stay.

Although teleconsultation may expedite care or obviate the need for formal in-person consultation, many medical complaints in the ED do not require referral to a specialist for management. In these cases, point-of-care medical photography can aid in documentation and facilitate continuity of care. The utility of photography for documentation in the absence of specialist consultation should not be underestimated. Photography allows providers to fully capture the full extent of findings quickly and easily without the need for lengthy written descriptions that lack richness and specificity that photographs offer. More importantly, in a specialty where post-ED discharge care-be it in the hospital or as an outpatient-is crucial, photography creates a shared mental model among all of the providers who provide continuity of care for patients and allows progression or resolution of findings to be objectively tracked over time across multiple providers. Indeed, we observed that photographs were often referred to in subsequent outpatient notes and many patients were re-photographed within 2 weeks of their ED encounter. Patient surveys conducted at our institution (unpublished) reflected that patients also found photographs helpful for tracking progression of findings themselves.

\section{Legal Considerations}

The practice of point-of-care medical photography raises several legal questions and considerations. Although a full legal discussion is out of the scope of this manuscript, a few points are worth noting. In an environment like the ED where the risk of malpractice litigation is high, it is possible that photography may reduce legal exposure by providing clear and irrefutable documentation of findings as they appeared during the visit.

Another legal consideration includes procedures for obtaining and documenting informed consent. Consent can be written, verbal, or implied. Written consent can be obtained by asking the patient to sign a form providing permission to capture photographs. Verbal consent may be obtained by explaining the process for capturing photographs and how the photographs will be used, and the patient may provide consent by replying "Yes, I consent." Finally, implied consent may be obtained if a patient poses for a photograph, analogous to a patient consenting to having blood drawn by rolling up her sleeve and extending her arm to a phlebotomist. At Mayo Clinic, institutional policies require consent to be obtained using one of the former two methods (i.e., written or verbal).

We observed that consent for photography was poorly documented by emergency medicine providers. Because the PhotoExam application includes a two-step hard stop process requiring attestation that consent has been obtained, we are confident that the finding of low rates of consent documentation does not equate to low rates of informed consent. However, documentation of consent, including the mechanism (i.e., written, verbal) of consent, the person providing consent (e.g., patient, parent, caregiver), and the ways in which use of the photographs is consented (e.g., clinical care, medical education) are important to document.

One plausible explanation for low consent documentation rates is that providers may have thought their attestation at the two-step hard stop within the application served as sufficient documentation. An alternative explanation is that providers may have forgotten to document this consent in the time-pressured environment of the ED or thought that 
specific documentation of consent was not required as a routine part of medical care.

Current efforts at our institution are underway to update our medical photography consent process to address these challenges. One option is to include prompts within the application which ask the user whether consent was obtained in written or verbal form and from whom it was obtained. Such a mechanism may permit documentation of consent exclusively from within the application which can then be stored as part of the image metadata. Another potential way the barriers to documenting consent could be overcome is to delegate the process for obtaining consent and capturing photographs to other staff members (e.g., nurse, clinical assistant).

Finally, integrated point-of-care medical photography applications may facilitate compliance with privacy laws and regulations. In the United States, HIPAA requires that patients have access to a "designated record set" which includes "Other records that are used, in whole or in part, by or for the covered entity to make decisions about individuals." ${ }^{20}$ If photographs that were used in the course of clinical decision-making are not available within the EHR because they were stored solely on a health care provider's mobile device, institutions may be noncompliant with recordkeeping requirements of HIPAA. Furthermore, if patient photographs are taken using a personal mobile device which lacks security safeguards, providers may be in violation of the HIPAA security rule.

\section{Other Uses for Point-of-Care Medical Photography}

Finally, one key clinical scenario where point-of-care medical photography can be useful in the pediatric ED, but which was not addressed in our study, is for documentation of suspected abuse or neglect of a child. Institutional policies at our institution encourage the use of on-call professional medical photographers to document cases of suspected abuse or neglect of a child. This process ensures that high-quality photographs are taken according to best-practices and also allows for viewing of the images within the EHR to be restricted. At other institutions without access to on-call professional medical photographers, point-of-care photography applications may facilitate objective documentation of suspected abuse or neglect of a child. Institutions that utilize mobile point-of-care medical photography applications for this purpose may wish to provide additional training to ensure photographs are taken according to best-practices.

\section{Strengths and Limitations}

Strengths of this study include that it is the first to comprehensively summarize uses of point-of-care medical photography using a mobile device in the emergency medicine setting. Although we previously published ${ }^{14}$ on the enterprise-wide experience of medical photography at Mayo Clinic, this previous study was limited to analysis of aggregated meta-data, quality assessment for a small subset, and assessment of change in the frequency of calls to traditional medical photographers. Our study on use in primary care considered the application's use for assessment of dermato- logic findings and only looked at the outcomes of consultation (teleconsultation and in-person consultation) and biopsy. ${ }^{15}$ To this end, we did not assess the actual content of photographs, the documented rationale for capturing photographs, or other important downstream outcomes.

Another strength of this study is that we utilized two reviewers to extract data from the medical record and measured inter-rater agreement and reliability on a subset before proceeding with review of the remaining records. We also included a reasonably large number of clinical encounters which reflect a diversity of chief complaints seen in the pediatric ED.

This study is limited in that it analyzed only a subset of patients (i.e., pediatric) seen in a single setting at a single institution (i.e., ED at one large academic medical center). We chose to study use in the ED because we hypothesized that photographs from this setting would represent a relatively wide diversity of findings and uses of photographs. We subsequently chose to focus on pediatric patients to limit the records to a feasible subset for manual analysis and to focus on the authors' primary domain of expertise. Additional analyses within other departments (e.g., dermatology, orthopedic surgery, ophthalmology), populations (e.g., adult), and geographic locations (e.g., regional health system site) may reveal different use practices that reflect local workflows.

The retrospective nature of the study also meant that the purposes for performing medical photography could not always be unambiguously determined. In many cases, the reviewers had to infer the purpose of medical photography based on contextual clues within clinical documentation but could decline to indicate the purpose of medical photography if it could not be determined with a high level of confidence. Furthermore, we were limited in our ability to draw causeand-effect conclusions when considering outcomes that occurred following photography. While our anecdotal experience suggests that point-of-care medical photography expedites patient care, systematically-generated evidence to robustly support this hypothesis is lacking.

Finally, we were unable to compare patients who were photographed with those who were not due to the lack of a suitable control group. For example, interesting hypotheses to test would include whether length of stay and disposition differ between patients who were or were not photographed for the same chief complaint. Although we considered a casecontrol method of study, the diversity of chief complaints, varying case complexity, and other factors that may affect a provider's decision to nonrandomly conduct photography made it challenging to confidently assign a truly similar group of matched controls to yield valid insights.

\section{Future Areas of Study}

In addition to health care providers, patients also increasingly own smartphones and capture photographs of medical findings. Patients and providers may wish to integrate patient-taken photographs into the EHR. The practice of patients or parents sending digital photographs to a health care provider has been reported in the setting of 
postoperative wound care, with one-fifth of cases where a digital image was sent resulting in clinical action (e.g., referral for in-person visit or antibiotic prescription). ${ }^{21}$ However, a key barrier was lack of a standardized process for collecting and integrating photographs into the EHR. ${ }^{21,22}$ The proprietary third-party EHR software in use at our institution allows patients to attach self-taken photographs to messages electronically sent to their health care provider. In this way, patients are able to add photographs to their own medical record. Other smartphone-compatible image-capture devices, such as otoscopes, are also marketed on the internet and may be used to integrate photographs to the EHR and facilitate telehealth.

\section{Conclusion}

Point-of-care medical photography is a powerful tool for high-fidelity documentation of the physical examination in the pediatric ED. It is used for a variety of purposes, and it appears to facilitate continuity of care across time and providers; however, consent procedures require standardization. Together, these results support ongoing support and maintenance of the application at our institution. For other institutions without similar capability, the results highlight the role of point-of-care medical photography in the EHR era and provide justification for the adoption of similar applications to facilitate multidisciplinary clinical care.

\section{Clinical Relevance Statement}

Point-of-care clinical photography with an EHR-integrated application has multiple advantages over the use of native camera applications. EHR-integrated applications can be used for clinical documentation of changes over time as well as store-and-forward telemedicine. Institutional processes for documentation of informed consent require standardization.

\section{Multiple Choice Questions}

1. What was the most commonly-documented use of pointof-care medical photography using a mobile application in the emergency department setting?

a. Formal telemedicine consultation.

b. Informal "curbside" telemedicine consultation.

c. To supplement clinical documentation.

d. To track changes over time.

Correct Answer: The correct answer is option c. To supplement clinical documentation. In the present study, $5 \%$ of the photographs were documented to be taken to track findings over time, $6 \%$ of the photographs were taken for formal or informal telemedicine, and 54\% were captured to supplement traditional textual documentation of clinical findings.

2. Which of the following aspects of photograph quality were most frequently deficient in photographs taken using a mobile application in the emergency department setting?

a. Clear demonstration of the area of interest.

b. Clear portrayal of size.

c. Image coloration.

d. Image sharpness.

Correct Answer: Although images were largely observed to be of sufficient quality for clinical purposes, two-thirds of the photographs were observed to be deficient in one of five aspects using a standardized quality rubric. The most commonly deficient aspect was portrayal of size, where $64 \%$ did not clearly portray size. Therefore, the correct answer is option b. Clear portrayal of size.

Protection of Human and Animal Subjects

This study was conducted in accordance with the Declaration of Helsinki and was approved by the Mayo Clinic Institutional Review Board.

Funding

This study was funded by the National Institutes of Health, (Grant/Award Number: “UL1TR002377”); Mayo Clinic Department of Emergency Medicine.

\section{Conflict of Interest}

The authors have no personal conflicts of interest to declare. However, all authors are either current or former employees (C.M.L., T.R.H.) or trainees (R.M.C., G.Y.K., K.D.W.) at Mayo Clinic. The PhotoExam application is institutional intellectual property of Mayo Clinic. The PhotoExam application is currently only used internally at Mayo Clinic and is not currently licensed outside of Mayo Clinic. Statistical analysis was funded by discretionary funds provided by the Mayo Clinic Department of Emergency Medicine. RedCap support was funded by the National Institute of Health (Grant UL1TR002377).

\section{References}

1 Wilson GM. Early photography, goitre, and James Inglis. BMJ 1973;2(5858):104-105

2 Cheung A, Al-Ausi M, Hathorn I, Hyam J, Jaye P. Patients' attitudes toward medical photography in the emergency department. Emerg Med J 2005;22(08):609

3 Sandler J, Gutierrez RJ, Murray A. Clinical photographs: the gold standard, an update. Prog Orthod 2012;13(03):296-303

4 Morse GA, Haque MS, Sharland MR, Burke FJ. The use of clinical photography by UK general dental practitioners. Br Dent J 2010; 208(01):E1

5 Aveta A, Filoni A, Persichetti P. Digital photography in plastic surgery: the importance of standardization in the era of medicolegal issues. Plast Reconstr Surg 2012;130(03):490e-491e

6 Kunde L, McMeniman E, Parker M. Clinical photography in dermatology: ethical and medico-legal considerations in the age of digital and smartphone technology. Australas J Dermatol 2013;54(03):192-197

7 Accetta JL, Schoenfeld J, Bitar C, Murina A. Smartphones in dermatology: acceptance of smartphone photography by the informed patient. Dermatol Surg 2019. Doi: 10.1097/ DSS.0000000000001976 
8 Chan N, Charette J, Dumestre DO, Fraulin FO. Should 'smart phones' be used for patient photography? Plast Surg (Oakv) 2016;24(01):32-34

9 Kameda-Smith MM, Iorio-Morin C, Winkler-Schwartz A, et al; Canadian Neurosurgery Research Collaborative (CNRC). Smartphone usage patterns by Canadian Neurosurgery Residents: a national cross-sectional survey. World Neurosurg 2018;111: e465-e470

10 Li MK, Howard DP, King R. "A picture tells a thousand words" smartphone-based secure clinical image transfer improves compliance in open fracture management. Injury 2019;50(07): 1284-1287

11 Services USDoHH. Summary of the HIPAA security rule. 2013. Available at: https://www.hhs.gov/hipaa/for-professionals/security/laws-regulations/index.html. Accessed August 9, 2019

12 Ai AC, Maloney FL, Hickman TT, Wilcox AR, Ramelson H, Wright A; The Use of Clinical Images in Electronic Medical Records. A picture is worth 1,000 words. Appl Clin Inform 2017;8(03):710-718

13 Landman A, Emani S, Carlile N, et al. A mobile app for securely capturing and transferring clinical images to the electronic health record: description and preliminary usability study. JMIR Mhealth Uhealth 2015;3(01):e1

14 Wyatt KD, Willaert BN, Pallagi PJ, Uribe RA, Yiannias JA, Hellmich TR. PhotoExam: adoption of an iOS-based clinical image capture application at Mayo Clinic. Int J Dermatol 2017;56(12):1359-1365
15 Pecina JL, Wyatt KD, Comfere NI, Bernard ME, North F. Uses of mobile device digital photography of dermatologic conditions in primary care. JMIR Mhealth Uhealth 2017;5(11):e165

16 Harris PA, Taylor R, Thielke R, Payne J, Gonzalez N, Conde JG. Research electronic data capture (REDCap)-a metadata-driven methodology and workflow process for providing translational research informatics support. J Biomed Inform 2009;42(02):377-381

17 Uebersax J. Raw agreement indices. 2018. Available at: https:// www.john-uebersax.com/stat/raw.htm. Accessed September 17, 2019

18 Kottner J, Audigé L, Brorson S, et al. Guidelines for reporting reliability and agreement studies (GRRAS) were proposed. JClin Epidemiol 2011;64(01):96-106

19 Viera AJ, Garrett JM. Understanding interobserver agreement: the kappa statistic. Fam Med 2005;37(05):360-363

20 Services USDoHH. Individuals' right under HIPAA to access their health information 45 CFR § 164.524. 2016. Available at: https:// www.hhs.gov/hipaa/for-professionals/privacy/guidance/access/ index.html. Accessed August 9, 2019

21 Abbott LM, Magnusson RS, Gibbs E, Smith SD. Smartphone use in dermatology for clinical photography and consultation: current practice and the law. Australas J Dermatol 2018;59(02):101-107

22 Miller MW, Ross RK, Voight C, et al. Patient-generated digital images after pediatric ambulatory surgery. Appl Clin Inform 2016;7(03):646-652 